\title{
Cardiac intensive care management of high-risk percutaneous coronary intervention using the venoarterial ECMO support
}

\author{
Marco Zuin ${ }^{1} \cdot$ Gianluca Rigatelli $^{2}$ (D) $\cdot$ Ramesh Daggubati $^{3}$ \\ Published online: 1 November 2019 \\ (C) Springer Science+Business Media, LLC, part of Springer Nature 2019
}

\begin{abstract}
The emerging concept of high-risk percutaneous coronary intervention (HR-PCI) has required the adoption of a multidisciplinary team approach. Venoarterial ECMO (VA-ECMO) has been introduced as a temporary mechanical circulatory support (MCS) for HR-PCI patients in order to provide an adequate systemic perfusion during the procedure. Both patient's complexity and technological evolutions have catalyzed the development of critical care cardiology; however, ECMO therapy faces several challenges. Indeed, the management of patients on ECMO remains complex; moreover, the lack of specific recommendation for HR-PCI patients further complicates the management of these patients. In this narrative review, we give a reappraisal for the management of HR-PCI patients supported with VA-ECMO according to the available data published in current literature.
\end{abstract}

Keywords Mechanical circulatory support $\cdot$ Intensive care unit $\cdot$ Shock $\cdot$ Critical care $\cdot$ Hemodynamic monitoring $\cdot$ High-risk PCI · VA-ECMO

$\begin{array}{ll}\text { Abbreviations } \\ \text { AMI } & \text { Acute myocardial infarction } \\ \text { CAD } & \text { Coronary artery disease } \\ \text { CCU } & \text { Cardiac care unit } \\ \text { HR-PCI } & \text { High-risk percutaneous coronary intervention } \\ \text { IABP } & \text { Intra-aortic balloon pump } \\ \text { LV } & \text { Left ventricle } \\ \text { MCS } & \text { Mechanical circulatory support } \\ \text { PCI } & \text { Percutaneous coronary intervention } \\ \text { pVAD } & \text { Percutaneous ventricle assists devices } \\ \text { VA-ECMO } & \text { Venoarterial ECMO }\end{array}$

All the authors equally contributed to the manuscript.

Gianluca Rigatelli

jackyheart71@yahoo.it

1 Faculty of Medicine, Section of Internal and Cardiopulmonary Medicine, University of Ferrara, Ferrara, Italy

2 Cardiovascular Diagnosis and Endoluminal Interventions Unit, Rovigo General Hospital, Rovigo, Italy

3 Division of Cardiology, Department of Medicine, NYU Winthrop Hospital, Mineola, NY, USA

\section{Introduction}

Percutaneous treatments of acute coronary artery disease (CAD) have drastically changed over the last two decades [1]. In fact, a huge amount of efforts has been made in order to achieve "equivalent" outcomes compared to those expected from the traditional by-pass surgery [2]. As result, percutaneous coronary interventions (PCIs) have become more complex and most frequently performed in patients with multiple comorbidities. Furthermore, an increasing number of patients not candidates for surgery due the higher pre-operative risk has been more frequently referred to PCI [3]. The complex peri-operative management of these subjects has required to adopt a collaborative team-based model. In this scenario, the term "high-risk PCI" (HR-PCI) has been recently coined to indicate a PCI performed providing short-term mechanical circulatory support (MCS) $[4$, 5]. Among these procedures, a growing number of PCIs have been performed using the venoarterial extracorporeal membrane oxygenation (VA-ECMO), both during rescue intervention in acute myocardial infarction (AMI) or elective HR-PCI, to maintain an adequate organ perfusion and prevent further hemodynamic deteriorations [6]. Despite the widespread diffusion of cardiac care unit (CCU) and even more sophisticated antiischemic therapies, a small, but not neglectable proportion of 
HR-PCI patients require VA-ECMO, hemodynamic and ventilatory support in cardiac care unit (CCU) [7]. In this narrative review, we give a reappraisal for the management of HR-PCI patients supported with VA-ECMO according to the available data published in medical literature.

\section{Definition of HR-PCI}

Nowadays, a clear definition of HR-PCI has not yet been provided since this clinical entity is constantly evolving. However, in 2015, Rihal et al. have identified some features able to predict a higher perioperative risk. These characteristics have been categorized into the following groups: (1) patient-specific, (2) lesion-specific, and (3) clinical presentation (Table 1; Fig. 1) [5].

Specifically, patient-specific factors include increased age (> 75 years old), diabetes mellitus (DM), relevant chronic kidney disease (CKD) or chronic obstructive pulmonary disease (COPD), severe heart valvular disease (HVD), heart failure $(\mathrm{HF})$, previous myocardial infarction (MI), peripheral artery disease (PAD), and previous history of transient ischemic attack (TIA) or stroke.

The lesion-specific group includes ostial stenosis, last patent conduits, presence of a severe multivessel coronary artery disease (defined as a SYNTAX score $>33[8,9]$ ), chronic total occlusions (CTOs), heavily calcified lesion, and significant stenosis involving the left main (LM) or its bifurcation.

Conversely, the group of items referred to clinical presentation refers to the hemodynamic status, left ventricular function, and presence or risk of electrical instability.

\section{Rationale of mechanical circulatory support during $\mathrm{HR}-\mathrm{PCl}$}

Physiologically, PCI systematically induces a transient myocardial ischemia, which is generally well tolerated in

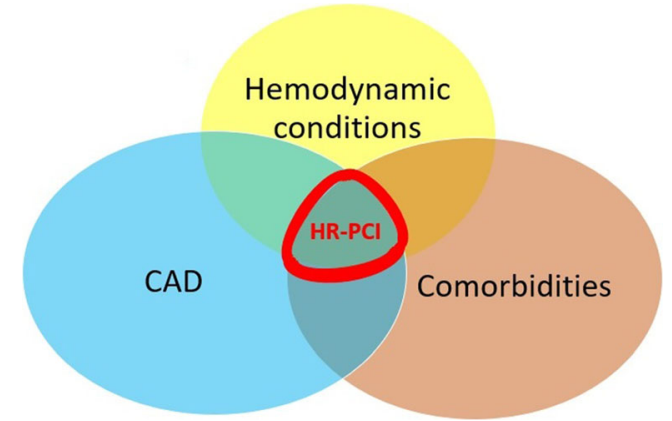

Fig. 1 Graphical representation of combined features related to the three main clinical areas currently defining high-risk PCI. CAD: coronary artery disease; HR-PCI: high-risk PCI

patients without significant comorbidities and/or impaired hemodynamic compensatory mechanisms. Conversely, those subjects with a basal poor left ventricular (LV) function, either for acute or chronic causes, multiple comorbidities, and/or severe CAD, cannot be able to face the transient PCI-related ischemia [10]. As consequence, a prophylactic cardiac assistance provides a more stable hemodynamic profile during both the procedure postoperative period.

\section{Interventional cardiologists and HR-PCI: when and why?}

It is not possible to plot all the potential scenarios in which interventional cardiologists can be involved in the support of HR-PCI patients. Indeed, critical care can be required both in the pre- and/or post-operative period. A protective PCI can be performed in high-risk patients without any complications and/or the need of postoperative advanced monitoring/support. At the same manner, patients admitted with ST-segment elevation myocardial infarction (STEMI) having multiple organ failure can require either a pre-operative hemodynamical stabilization
Table 1 Characteristics related to a higher peri-operative risk in patients undergoing percutaneous coronary intervention

\begin{tabular}{|c|c|c|}
\hline Patient-specific & Lesion-specific & Clinical presentation \\
\hline Age $>75$ years & Ostial stenosis & Hemodynamic status \\
\hline Diabetes mellitus & Last patent conduits & Left ventricular function \\
\hline Chronic kidney disease & $\begin{array}{l}\text { Severe multivessel coronary artery disease } \\
\quad(\text { SYNTAX score }>33)\end{array}$ & $\begin{array}{l}\text { Arrhythmias or electrical } \\
\text { instability }\end{array}$ \\
\hline $\begin{array}{l}\text { Chronic obstructive pulmonary } \\
\text { disease }\end{array}$ & Chronic total occlusions & \\
\hline Severe heart valvular disease & Heavily calcified lesion & \\
\hline Heart failure & Significant stenosis involving the left main & \\
\hline Previous myocardial infarction & Left main bifurcation stenosis & \\
\hline \multicolumn{3}{|l|}{ Peripheral artery disease } \\
\hline $\begin{array}{l}\text { Previous transient ischaemic } \\
\text { attack or stroke }\end{array}$ & & \\
\hline
\end{tabular}


followed by advanced life support during the postprocedural period in $\mathrm{CCU}$.

\section{Percutaneous devices for high-risk PCl: a brief overview}

The aim of short-term MCS in HR-PCI is to reduce both myocardial work and oxygen demand while maintaining adequate systemic and coronary perfusion [5]. From a theoretical point of view, the optimal device should be able to increase the mean arterial pressure (MAP), cardiac output $(\mathrm{CO})$, and coronary artery perfusion while unloading the left ventricle (LV) and reducing the cardiac work $(\mathrm{CW})$. In daily clinical practice, MCS has been provided using different temporary percutaneous devices, as the "traditional" intra-aortic balloon pump (IABP), the Impella (ABIOMED Inc, Danvers, Massachusetts, US) or the TandemHeart (Cardiac Assist Inc, Pittsburgh, Pennsylvania, US) platforms, which are frequently named percutaneous ventricle assist devices (pVAD), or the VAECMO. These devices can provide different types of MCS, for different periods of time and clinical scenarios. Furthermore, the VA-ECMO, due to its properties, is generally used in combination with other MCS devices. Despite the description of their properties is out of the aim of the present review, a brief comparison of these devices is given in Table 2 .

In everyday practice, the real challenge remains to choose which device offers the most adequate support in each patient. Generally, in patients with pre-shock (defined as a systemic hypoperfusion with a systolic blood pressure $<100 \mathrm{mmHg}$ ), hemodynamic support is generally provided using intra-aortic balloon counterpulsation (IABC) [11]. Conversely, patients with severe CS should receive a pVAD as first-line treatment, while, in case of further clinical deterioration such as refractory CS shock or cardiac arrest supported with cardiopulmonary resuscitation after an acute myocardial infarction (AMI), the MCS is further escalated to VA-ECMO (Fig. 2) [12].

\section{VA-ECMO properties}

VA-ECMO provides excellent circulatory support and blood oxygenation also in HR-PCI patients. Most of the available data on the use of VA-ECMO in these groups of patients have been obtained by single-center, observational, or single-series studies [13]. Unfortunately, VA-ECMO is unable to effectively unload the left ventricle (LV) since it increases both the afterload and thereby the myocardial oxygen consumption and myocardial work [14]. Furthermore, a higher afterload progressively leads to
LV distension, intracardiac thrombosis, pulmonary edema, and hemorrhage, which represents some of the potential adverse events related to the VA-ECMO use. To prevent these hemodynamic unfavorable effects, VA-ECMO is generally used in combination with other devices for MCS such as Impella or IABC.

Different cannulation strategies can be adopted for VAECMO patients. Among these, the central cannulation remains the method of choice for patients with shock postcardiotomy. Conversely, the peripheral cannulation currently represents the most commonly applied approach. Generally, the right femoral vein is used as venous cannulation with its tip lying in the right atrium (RA). Instead, for the arterial cannulation, the contralateral side is adopted. Moreover, in recent years, also, an upper extremity cannulation has been also adopted using an internal jugular venous and axillary artery arterial cannulas [15].

\section{VA-ECMO implantation}

VA-ECMO can provide full hemodynamic support, independently from the underlying heart rhythm, simultaneously reducing carbon dioxide $\left(\mathrm{CO}_{2}\right)$ and adding oxygen $\left(\mathrm{O}_{2}\right)$ to venous blood before returning to the arterial circulation, bypassing pulmonary circulation. VA-ECMO insertion can be performed using central or peripheral cannulation. In the setting of HR-PCI, the peripheral approach is generally preferred since it allows an easier cannulation and decannulation. Specifically, venous cannulation can be performed using both an infrahepatic inferior vena cava cannula or a large 21-25 French cannula inserted in the femoral vein with its tip in the RA. Conversely, oxygenated blood is returned via the arterial cannula (17-21 French) inserted in the femoral artery. The tip of the venous and arterial cannula end into the right atrium (RA) and in the iliac artery, respectively [6].

\section{Use and potential complications of VA-ECMO}

Peripheral cannulation for the use of VA-ECMO can be also performed at bedside without the need of fluoroscopy or echocardiography. However, the larger part of VAECMO implantation is performed in cath-lab because these patients frequently had arterial and/or venous occlusive disease. Despite that the platform is completely transportable, cannula misplacement and/or dislodgments can be carefully avoided, especially during the patients' mobilization.

Among the device-related complications, vascular injuries, due to the large cannula size, bleeding events, 
Table 2 Comparison of different mechanical circulatory support devices (MCS) used in high-risk percutaneous coronary intervention

\begin{tabular}{|c|c|c|c|c|}
\hline & IABP & IMPELLA & TANDEMHEART & VA-ECMO \\
\hline & & 2.5 & & \\
\hline \multicolumn{5}{|l|}{ General features } \\
\hline Cardiac flow (L/min) & $0.3-05$ & 1.5 & $2.5-5$ & $3-7$ \\
\hline Mechanism & Aorta & Left ventricle $\rightarrow$ Aorta & Left atrium $\rightarrow$ Aorta & Right atrium $\rightarrow$ Aorta \\
\hline $\begin{array}{l}\text { Cardiac Synchrony or stable } \\
\text { rhythm }\end{array}$ & Yes & No & No & No \\
\hline Implant days & Weeks & 7 days & 14 days & Weeks \\
\hline \multicolumn{5}{|l|}{ Hemodynamic properties } \\
\hline MAP & $\uparrow$ & 个个 & 个个 & 个个 \\
\hline Afterload & $\downarrow$ & $\downarrow$ & $\uparrow$ & 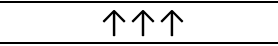 \\
\hline LVEDP & $\downarrow$ & $\downarrow \downarrow$ & $\downarrow \downarrow$ & $\leftrightarrow$ \\
\hline PCWP & $\downarrow$ & $\downarrow \downarrow$ & $\downarrow \downarrow$ & $\leftrightarrow$ \\
\hline LV Preload & - & $\downarrow \downarrow$ & $\downarrow \downarrow$ & $\leftrightarrow$ \\
\hline Coronary artery perfusion & $\uparrow$ & $\uparrow$ & - & - \\
\hline Myocardial oxygen demand & $\downarrow$ & $\downarrow \downarrow$ & $\downarrow$ & $\leftrightarrow$ \\
\hline \multicolumn{5}{|l|}{ Contraindications } \\
\hline Valve disease & $\begin{array}{c}\text { Moderate to severe } \\
\text { AR }\end{array}$ & Moderate to severe AR & $\begin{array}{c}\text { Moderate to } \\
\text { severe AR }\end{array}$ & $\begin{array}{c}\text { Moderate to severe } \\
\text { AR }\end{array}$ \\
\hline Aortic disease & Aortic disease & $\begin{array}{c}\text { Aortic Stenosis } \\
(\text { AVA <0.6) } \\
\text { Mechanical } \\
\text { aortic valve }\end{array}$ & & \\
\hline PAD & Severe PAD & Severe PAD & Severe PAD & \\
\hline Anticoagulation & & Contraindications to $\mathrm{AC}$ & $\begin{array}{l}\text { Contraindications } \\
\text { to } A C\end{array}$ & $\begin{array}{c}\text { Contraindications to } \\
\text { AC }\end{array}$ \\
\hline Intracardiac thrombosis & & LV thrombus & LA Thrombus & \\
\hline \multirow[t]{2}{*}{ Coagulation disorders } & & & HIT & \\
\hline & & & DIC & \\
\hline Septal defects & & & VSD & \\
\hline \multicolumn{5}{|l|}{ Complications } \\
\hline Stroke & $\mathrm{X}$ & $x$ & $x$ & \\
\hline Limb Ischemia & $x$ & $\mathrm{X}$ & $\mathrm{X}$ & $\mathrm{X}$ \\
\hline Vascular trauma & $\mathrm{X}$ & $\mathrm{x}$ & $x$ & $\mathrm{x}$ \\
\hline Infection & $\mathrm{X}$ & $\mathrm{X}$ & $\mathrm{X}$ & $x$ \\
\hline Haemolysis/ bleeding (risk) & $X(+)$ & $X(++)$ & $X(++)$ & $X(++)$ \\
\hline Device dislodgment/migration & $\mathrm{X}$ & $x$ & $x$ & \\
\hline $\mathrm{AKI}$ & $x$ & & & $\mathrm{x}$ \\
\hline Thromboembolism & & & $\mathrm{x}$ & $x$ \\
\hline Neurological injury & & & & $\mathrm{X}$ \\
\hline Device Thrombosis & & $x$ & & \\
\hline Compartment syndrome & & & & $x$ \\
\hline Balloon rupture & $\mathrm{x}$ & & & \\
\hline
\end{tabular}

hemolysis, infections, stroke, neurological or acute kidney injuries (AKIs), and pulmonary edema, are the most frequently observed. While the management of systemic complications can be managed by the interventional 


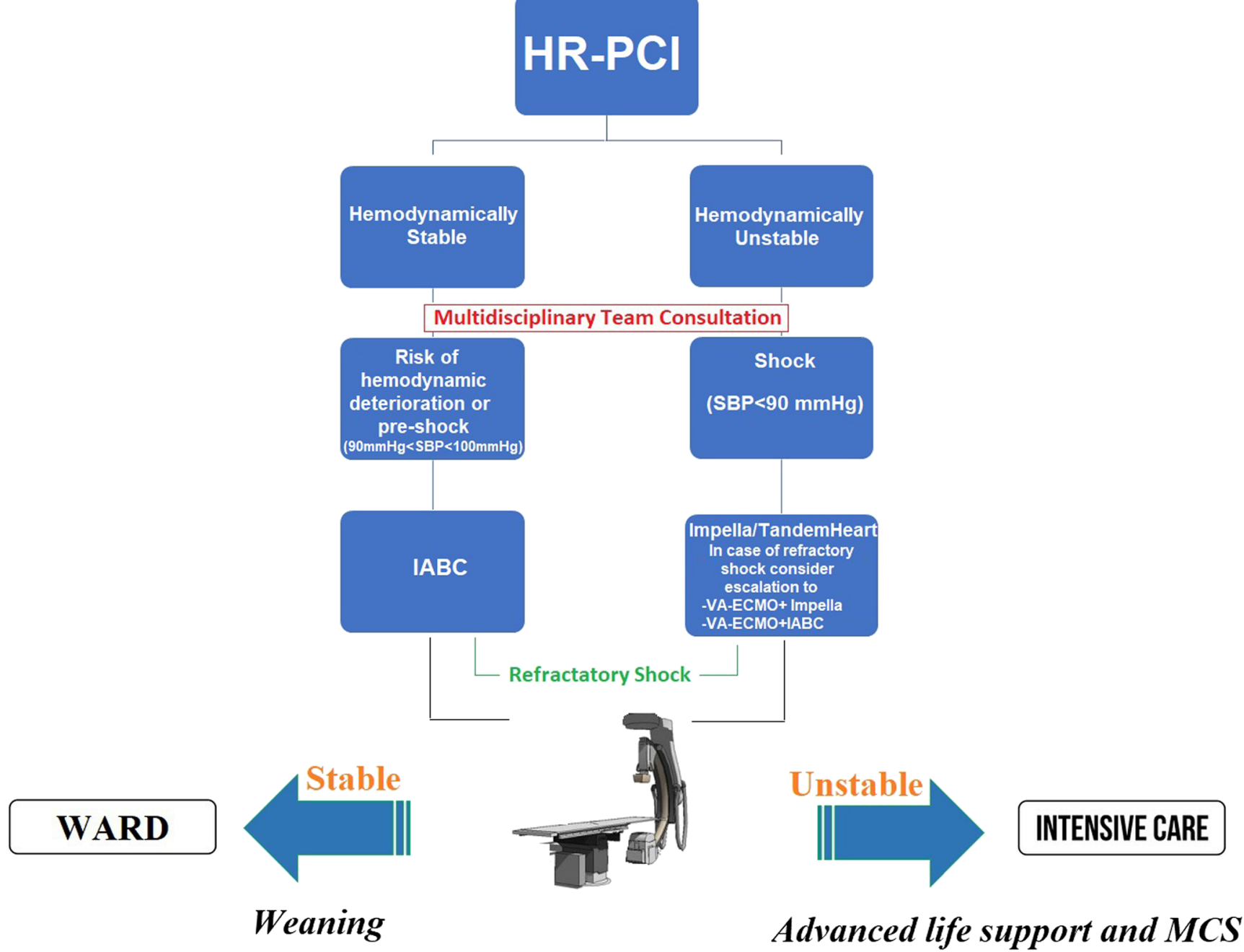

Fig. 2 Types of mechanical circulatory support provided accordingly to the baseline patient's hemodynamic status. SBP: systolic blood pressure; IABP: intra-aortic balloon-pump

cardiologists and/or intensivists, issues related to the cannulation require the coordination with the interventional cardiologist.

\section{Current evidences supporting the use of VA-ECMO in high-risk PCI}

\section{General aspects}

Shaukat et al. described the use of VA-ECMO in five patients treated with elective high-risk PCI with ECMO support. In all cases, MCS was successfully weaned within $24 \mathrm{~h}$ without any post-procedural complication or major adverse cardiovascular events (MACE) within 1 year [16]. Similarly, the reduced incidence of MACE and cerebral events as well as the more favorable short-term outcome in HR-PCI patients supported by VA-ECMO has been reported by Tommasello et al. [13]. Huang et al. successfully demonstrated that prophylactic
ECMO implantation in STEMI patients with refractory CS significantly improved both short- and long-term outcomes [17].

\section{Concurrent implantation of VA-ECMO and Impella: "EC-PELLA"}

Patients supported with VA-ECMO need to be unloaded to prevent a failing static LV. In this regard, different investigations have demonstrated that the concomitant implantation of the Impella system in these patients (so-called "EC-PELLA") is a viable solution. Patel et al. analyzed the largest US-based dataset on the use of VA-ECMO, with and without simultaneous Impella support, in patients with refractory CS. They demonstrated that the addition of the Impella system was significantly associated with a lower 30-day all-cause mortality as well as lower need for inotropic support and a comparable safety profile as compared with VA-ECMO alone [18]. Similar findings were reported by Pappalardo et al. in a 2:1 
propensity-matching analysis based on the comparison between patients treated with and without the combination of VA-ECMO and Impella®. They demonstrated that patients in the VA-ECMO and Impella group had a significantly lower hospital mortality ( $47 \%$ vs. $80 \%, P<0.001$ ), higher rate of successful bridging to either recovery or further therapy $(68 \%$ vs. $28 \%, P<0.001)$ compared with VA-ECMO patients. No significant differences in the occurrence of major bleeding events were observed among the two groups $(p=0.6)$ [19]. Akanni et al. recently reported in a retrospective analysis comparing patients treated with pLAVD or only VA-ECMO therapy that despite a higher rate of hemolysis in the former ( $44.83 \%$ vs $17.35 \% p=0.002$ ), the combined use of VAECMO and pLVADP may improve or circumvent LV distension in refractory CS [20].

\section{Concurrent implantation of VA-ECMO and intra-aortic balloon pump}

Despite used less frequently, the combination of a VA-ECMO plus IABP has demonstrated to effectively unload the LV by different clinical investigations (Fig. 3) [21]. Li et al. compared the effect of VA-ECMO plus IABP with that of VAECMO alone in a meta-analysis based on 29 studies. These authors reported that VA-ECMO plus IABP was associated with decreased in-hospital deaths [risk ratio (RR) $0.90 ; 95 \%$ confidence interval (CI) $0.85-0.95 ; P<0.0001]$. Furthermore, IABC was associated to decreased in-hospital mortality of patients with extracorporeal cardiopulmonary resuscitation, post-cardiotomy CS, and ischemic heart disease. Intriguingly, gastrointestinal, neurological, and limb-related were comparable between the two groups [22].

\section{Tandem heart, Maquet Cardiohelp, and VA-ECMO}

The TandemHeart ${ }^{\circledR}$ device (CardiacAssist Inc.) is an external centrifugal pump that can be used in HR-PCI patients. This
MCS device consists in a 21-Fr inflow cannula placed transeptally into the LA and an outflow cannula placed into the femoral artery. Cannulation requires to be done in the Cath lab using fluoroscopy and echo guidance. However, the need for transeptal puncture has and still represents the major limitation for operators [23]. Other investigations have reported that an adequate hemodynamic support in HR-PCI patients can be rapidly achieved with excellent procedural success [24]. Negi et al. have compared the TandemHeart and VAECMO in patients with AMI and refractory CS demonstrating a no significant differences in survival rate as well as in the incidence of complications such as limb ischemia, significant hemolysis, need for renal replacement therapy (RCT), stroke or recurrent myocardial infarction between the two groups. However, a higher incidence of ventricular arrhythmic events was registered in VA-ECMO patients $(16 \%$ vs. $50 \%, p=0.02)$ [25]. Similarly, Chamogeorgakis et al. demonstrated no differences in the short-term mortality in CS patients supported with VA-ECMO and TandemHeart or Impella [26]. Moreover, Bernhardt et al. have demonstrated that the use of TandemHeart in an ECMO represents a feasible and sage prevention strategy for pulmonary edema and an adjunctive treatment able to facilitate the weaning from VA-ECMO in patients with CS and LV thrombus [27]. As for the Impella, limb ischemia, hemolysis, vascular trauma, thromboembolism, and cardiac tamponade are potential life-threating complications related to the use of this MCS device.

Some interesting data has been presented also using the Maquet Cardiohelp (MAQUET Cardiopulmonary AG; Germany). This device is currently the world's smallest portable heart-lung support system, available mostly for ECMO patients who need transportation. The Maquet Cardiohelp is able to provide several clinical information such as system pressures, mixed venous oxygen saturation, and hematocrit utilizing the incorporated sensors. Its use in the setting of HR-PCI has been successfully presented by different investigations [28-30].
Fig. 3 a An example of patient with complex PCI and high-risk features assisted by IABP + Impella. b Mechanical circulatory support provided by IABP plus VA-ECMO. The position of ECMO's venoarterial cannulas, inserted percutaneously, is highlighted into the magnification
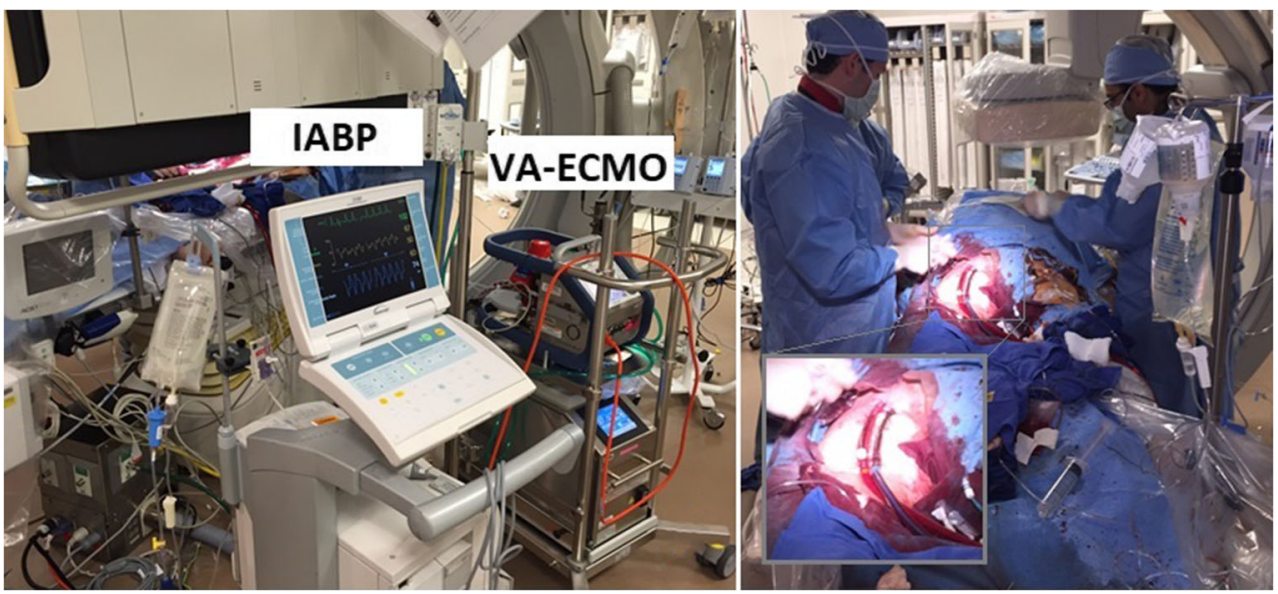


\section{Intensive care monitoring and weaning from VA-ECMO}

Hemodynamic monitoring is central in the management of ICU patients, especially in those receiving MCS. Adequate monitoring plays a pivotal role in assessing the course of the underlying life-threatening cardiac and/ or respiratory diseases. Moreover, its appropriate use remains fundamental to plan the timing of weaning from the MCS. The weaning planification must carefully outweigh the risk of mortality or adverse events towards the myocardial recovery. Two different groups of issues should be considered: patient- and device-related monitoring (Table 3) [31]. Volume status, degree of LV pressure unloading, mean arterial pressure (MAP), central venal pressure, and PCWP are essential to maintain or add additional LV unload. Most of HR-PCI patients require additional vasopressor support which must be tailored only after considering the patient's volume status (Fig. 4). As evidenced in Table 3, bedside transthoracic echocardiography (TTE) plays a fundamental role not only for monitoring the clinical course of the underline ischemic cardiomyopathy but also to detect complications and support the decision making. Conversely, different investigations have demonstrated that cardiac biomarkers are not useful for identifying those who will recover [32].

To date, only few investigations have analyzed potential strategies for weaning from VA ECMO [33-35]. To over-complicate this matter, the percentage of patients successfully weaned from ECMO varies from 31 to $76 \%$, depending on the underlying cause of CS and the definition of successful weaning [36]. Moreover, weaning does not mean survival, because up to $65 \%$ of patients weaned from VA ECMO support do not survive to hospital discharge $[37,38]$. Current recommendations and TTE criteria for the weaning from VA-ECMO are resumed in Fig. 5a, b, respectively. It is useless to attempt weaning within the first $72 \mathrm{~h}$ after VA ECMO implantation, because damaged organs need time to recover. Previous investigations have reported that patients could be considered for VA ECMO weaning when the following hemodynamic parameters are maintained with the pump off: cardiac index $>2.4 \mathrm{~L} / \mathrm{min} / \mathrm{m}^{2}$, MAP $>60 \mathrm{mmHg}$, pulmonary capillary wedge pressure $<18 \mathrm{~mm} \mathrm{Hg}$, and central venous pressure $<18 \mathrm{mmHg}[38,39]$.

Previous efforts, made to identify potential predictors of successful weaning from VA-ECMO, have demonstrated that only the pulse pressure appeared to be unquestioned clinical factor associated with weaning success $[33,40,41]$. Further studies are needed to recognize potential markers of successful weaning from VA-ECMO, especially in those requiring a relative short MCS, such as HR-PCI patients.

\section{VA-ECMO and the right ventricle}

As known, the RV is a thin-walled and compliant chamber which has received less attention over the years when compared to LV. As a matter of fact, nowadays, no clinical trials have investigated the effect of VA-ECMO alone in the treatment of acute RV failure despite several investigations have reported the efficacy and feasibility of this MCS in these patients [42]. However, despite VA-ECMO results not able to intrinsically unload the LV, especially when the LV function is severely compromised, it is able to efficiently unload, when used alone, both the right atrium (RA) and ventricle (RV) by decreasing the right ventricular preload [43]. Unfortunately, the assessment of the RV function during full VA-ECMO support remains difficult because the ECMO circuit creates negative pressure and drains venous blood from the RA [37].

Determination of the left ventricular (LV) and right ventricular (RV) function before ECMO weaning remains essential to predict biventricular or univentricular recovery. Indeed, lack of recognition of significant coexisting RV dysfunction may significantly increase the post-procedural morbidity and mortality as well as requiring prolonged inotropic agents, biventricular device support, or prolonged extracorporeal support [34]. Moreover, animal models have demonstrated that the unloaded RV during VA-ECMO support has a lower metabolic flexibility contributing to the inability to increase highenergy phosphate reserve during MCS [44]. In this setting, adjuvant RV support during weaning can be required. Surely, further translational and clinical studies are needed to further optimize the management of the RV in patients requiring VA-ECMO support, especially after HR-PCI.

\section{Post PCI management}

From a theoretical point of view, VA-ECMO and other types of MCS should be removed at the end of the HR-PCI in cathlab unless the weaning cannot be performed. In this regard, no specific criteria still exists, and in most of the case, a patienttailored approach is generally performed. However, some generic criteria, based in part on those used for the standard weaning from VA-ECMO, should be used to determine in which patients the MCS can be stopped at the end of coronary artery procedure. During the weaning trial, if the patient maintains a $\mathrm{MAP} \geq 65 \mathrm{mmHg}$, a CVP $<15 \mathrm{mmHg}$, a LVEF $\geq 25 \%$, in the absence of LV or RV dysfunction and preserved respiratory function (evaluated in terms of $\mathrm{FiO} 2$ and $\mathrm{PaO} 210 \mathrm{~min}$ after having decrease the ECMO and sweep gas flows), absence of intra-procedural complication and a successful revascularization defined and a TIMI 3 flow in all treated lesions, physicians can consider to stop the MCS directly in the cathlab. However, in the presence of significant comorbidities (either cardiac, respiratory, or metabolic) as well as in patients which had a higher probability of early clinical 
Table 3 Patient- and device-related issues requiring monitoring in high-risk percutaneous coronary intervention patients supported with VA-ECMO

\begin{tabular}{|c|c|c|c|}
\hline Patient-related issues & Parameters & $\begin{array}{l}\text { Useful to plan and evaluate } \\
\text { the weaning phase }\end{array}$ & TTE \\
\hline \multicolumn{4}{|l|}{ Hemodynamic issues } \\
\hline \multirow[t]{3}{*}{ LV load } & LVEDD & & $*$ \\
\hline & Grade of MR & & $*$ \\
\hline & PCWP & & \\
\hline \multirow{2}{*}{$\begin{array}{l}\text { LV inotropic-afterload rela- } \\
\text { tion }\end{array}$} & Pulsatility of arterial blood pressure & & \\
\hline & Opening of the aortic valve & & $*$ \\
\hline RV preload & Size of the RA & $*$ & $*$ \\
\hline Volume status & CVP & & \\
\hline LV-inotropy & LVEF & $*$ & $*$ \\
\hline RV-inotropy & RVEF; TAPSE & $*$ & $*$ \\
\hline LV-preload & PCWP & $*$ & $*$ \\
\hline LV-afterload & MAP & $*$ & \\
\hline Heart rate: & Continuous ECG & & \\
\hline Microcirculation & Serum lactate & * & \\
\hline Cardiac output & Central venous $\mathrm{O} 2$-saturation & * & \\
\hline \multicolumn{4}{|l|}{ Perfusion } \\
\hline Leg ischemia & Clinical signs & $*$ & \\
\hline Peripheral ischemia & Serum lactate & $*$ & \\
\hline Cardiac ischemia & $\mathrm{cTn}$ & $*$ & \\
\hline \multicolumn{4}{|l|}{ Ventilation and gas exchange } \\
\hline $\begin{array}{l}\text { Adequate gas exchange and } \\
\text { prevention of VILI }\end{array}$ & $\begin{array}{l}\text { ABG; pulse oximetry; lung imaging (either } \\
\text { x-ray or US) }\end{array}$ & $*$ & \\
\hline $\begin{array}{l}\text { Anticoagulation status and } \\
\text { prevention of haemolysis }\end{array}$ & $\begin{array}{l}\text { ACT, fibrinogen, platelets, } \mathrm{LDH} \text {, haptoglobin, } \\
\text { indirect bilirubin, reticulocytes }\end{array}$ & & \\
\hline $\begin{array}{l}\text { Monitoring of underling } \\
\text { disease or patient's general } \\
\text { assessment }\end{array}$ & Routine laboratory tests & & \\
\hline \multicolumn{4}{|l|}{ Device-related issues } \\
\hline $\begin{array}{l}\text { Bleeding, infections at the } \\
\text { cannula site insertion }\end{array}$ & Inspection & & \\
\hline $\begin{array}{l}\text { Cannula dislodgment at the } \\
\text { insertion site }\end{array}$ & Inspection & & \\
\hline Pump function & $\begin{array}{l}\text { Pump driving speed, pump minute volume, } \\
\text { Temperature, FiO2, arterial pressure, venous } \\
\text { suck pressure }\end{array}$ & $*$ & \\
\hline
\end{tabular}

$L V$ left ventricle, $R V$ right ventricle, $L V E D D$ left ventricular end diastolic diameter, $M R$ mitral regurgitation, $P C W P$ pulmonary capillary wedge pressure, $R A$ right atrium, $C V P$ central venous pressure, $L V E F$ left ventricular ejection fraction, $R V E F$ right ventricular ejection fraction, TAPSE tricuspid annular plane systolic excursion, MAP mean arterial pressure, $c T n$ cardiac troponin, $A B G$ arterial blood gas analysis, $L D H$ lactate dehydrogenases, $T T E$ transthoracic echocardiography

decompensation due to the type of the lesion treated (last conduit, left main, or left main bifurcation) or have experienced significant arrhythmic event during the PCI, the weaning from the MCS should be delayed [39, 40].

After the PCI, a more intensive and medical and nursing cares are required for these patients. Several problems can be encountered during VA-ECMO support (Table 4). In this regard, vascular access and limb perfusion must be carefully monitored. Cannula dressings may be performed by nursing staff following consultation with the interventional cardiologist or the ICU physician. A standard invasive line dressing procedure should be used without using alcohol-based solutions but applying betadine and subsequently covering with an occlusive and transparent dressing. During the procedure, the sterility of the operator must be guaranteed.

Prevention of limb ischemia represents another fundamental aspect of the post-operative management. A recent metaanalysis has reported that this complication occurs in about $17 \%$ of VA-ECMO patients while among these, $10 \%$ progress to compartment syndrome and $4.7 \%$ to amputation [45]. To avoid these events, some techniques can be adopted to improve the distal arterial flow in the limbs. A distal perfusion 


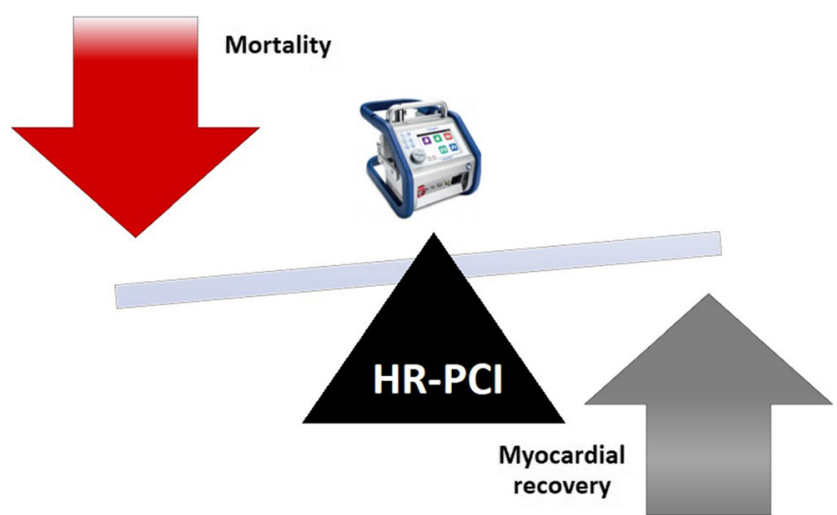

Fig. 4 VA-ECMO weaning planification must carefully outweigh the risk of mortality or adverse events towards the myocardial recovery

cannula (DPC) could be inserted distally to the arterial return cannula or a T-graft can be performed in place of a direct arterial cannulation, but this last one approach required a surgical approach which can lead to the hyperemia of the limb [46, 47].

A major disadvantage of peripheral cannulation is represented by the risk of the so-called Harlequin or North-South syndrome. This event is observed as a consequence of upper body hypoxemia which can be due to the concomitant lung failure or wrong ventilator settings. From a pathophysiological point of view, this event occurs when well-oxygenated retrograde blood from the femoral arterial cannula meets poorly oxygenated blood from the LV. In these patients, the blood perfusing the brain, the heart, and the upper extremities may have a saturation below the $90 \%$. After a prompt recognition, ventilator setting must be adjusted to improve the oxygenation of pulmonary venous return or by decreasing the LVEF. Alternatively, a V-VA ECMO should be considered [15].

Always to prevent bleeding and thrombotic events, an adequate anticoagulation must be maintained. Nowadays, anticoagulation therapy in HR-PCI patients is adjusted accordingly to the activated clotting time (ACT). Unfractionated heparin (UFH) currently remains the main anticoagulant in VAECMO patients after a HR-PCI due to its rapid onset and potential neutralization with protamine sulfate. Indeed, these patients are generally treated also with antiplatelet drugs due to the recent PCI. It is easy to imagine that the bleeding risk and the occurrence of bleeding events often happen. In some patients, the administration of UFH may cause a heparin-induced thrombocytopenia (HIT) which must be promptly recognized and managed modifying the anticoagulation strategies. In this scenario, thrombin inhibitors (e.g., Bivalirudin or Argatroban) must be administered; these drugs are independent from the antithrombin levels , have no antagonists and a lower coagulative inhibition in area of stasis $[48,49]$.

Different alarms can be set on the ECMO console. In this regard, it is important to consider that when an alarm is set on, the VA-ECMO stop working and an immediate must be set to resolve the problem. The continuous presence of a bedside or ICU perfusionist should be highly due to the profound impact on the patient's care. However, the perfusion services must be contacted regarding all circuit issues related to VA-ECMO and the contact information for the perfusionist-on-call must be provided at the bedside [50].

A real emergency is represented by the membrane oxygenator rupture. Small defects in the integrity of the blood/gas barrier with little bleeding can be tolerated because the small tear will usually clot off. However, major bleeding events require an emergent oxygenator change. Despite that it is a rare event, a massive air embolus and death can occur in this scenario.

A close collaboration between physicians, registered nurses (RNs), and perfusionists plays a pivotal role in the management of these complex patients. In this regard, an adequate and interdisciplinary training as well as shared operative protocols are required.

\section{Mechanical ventilation}

Patients with a low $\mathrm{CO}$ are generally predisposed to decrease pulmonary compliance, to V/Q mismatch and premature airway closure. Similarly, HR-PCI patients, especially those with

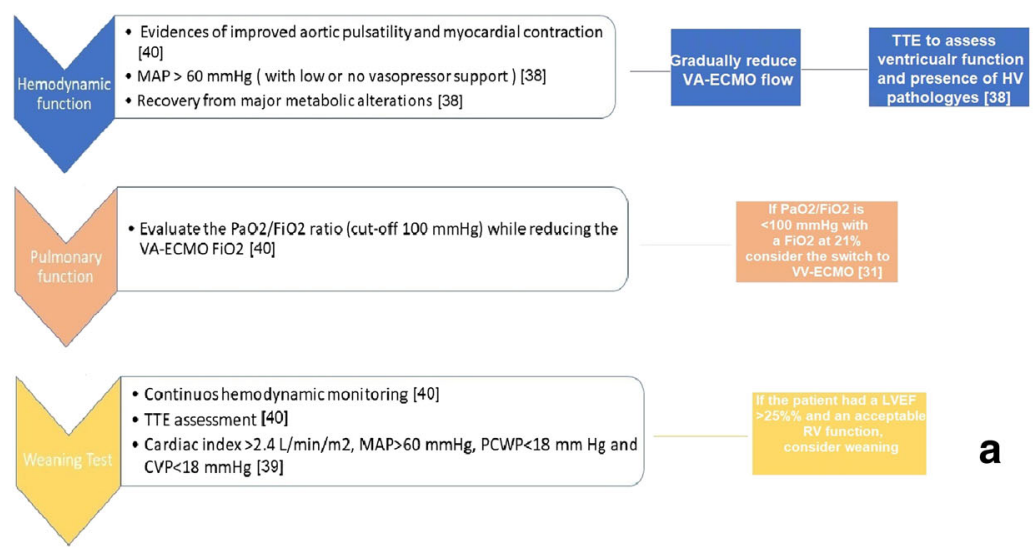

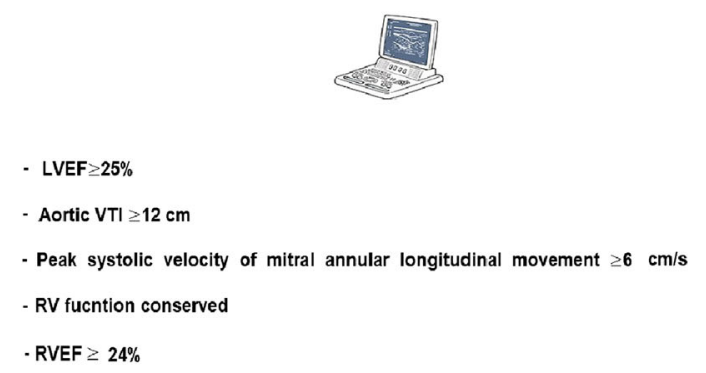

Fig. 5 a Current recommendations for the weaning from VA-ECMO obtained by reviewing the current literature. b Current echocardiographic criteria for VA-ECMO weaning 
Table 4 Common and life-threatening problems related to the use of VA-ECMO

Problems and potential causes Clinical presentation Actions

Common problems

Bleeding cannula site

Limb ischemia

-Arterial embolism

-Haematoma compression

-Large femoral arterial cannula (not allows retrograde perfusion)

Flow-fluctuation

-Problem with the venous access line

-Low venous pressure.

Circuit thrombosis

Decreased venous pressure

- Low intravascular volume

- Increased intrathoracic pressure

- Increased intraabdominal pressure

Severe problems

Motor failure

$\mathrm{AC}$ power failure

Pump head disengagement

Pump flow sensor failure

Accidental venous access decannulation

Accidental arterial access decannulation

Air embolism
Bleeding from the vascular access

Range from pain of different severity to pallor, poikilothermia, motor or sensory deficit and gangrene.

Values on VA-ECMO control panel

Related to the thrombotic burden

Values on VA-ECMO control panel

Resembles cardiac arrest.

Specific alarm can be present on the VA-ECMO console

Resembles cardiac arrest.

Resembles cardiac arrest.

an unusual grinding noise and vibration of the pump head should be perceived

Specific alarm on the VA-ECMO console

Bleeding loss from the cannulation site (in patient with central cannulation means blood loss from the RA.

Cardiac arrest

Cardiac arrest
-Confirm that the cannula is properly positioned.

-Thrombotic dressings

-Direct manual pressure (or with a sandbag)

-Decrease the ACT target

-Local injections of adrenaline $(1: 200,000)$ or adrenaline-soaked gauze

-Re-cannulation

-Perform a Doppler US of lower extremities Consult the vascular surgical team

-Assess the venous access line and correct the venous pressure if low

-Small clots in the oxygenator don't tend to modify the VA-ECMO function and can be monitored.

-Provide adequate anticoagulation

-Arrange staff and exchange the thrombosed circuit element.

-Correct the intravascular volume and/or modify the ventilation settings

-Hand-cranking the pump while getting a new motor console.

-Hand-cranking the pump while trying to re-establish AC power.

-Clamp the venous line

-Re-engage the pump head

-Turn the pump to a slow RPM setting (about 1000 RPM) and unclamp the line

-Gradually increase the RPM to the previous setting

-If the grinding sound persist the circuit needs to be changed urgently

-In the absence of clinical modification or sudden cardiac arrest the problem is due to the ultrasonic flow sensor. Contact the perfusionist

-Start CPR

-Control bleeding

-Re-establish the vascular access

-Obtain another circuit

-Clamp immediately the line

-Stop the pump

-Start CPR.

-Control bleeding: (in case of peripheral cannulation attempt to control the bleeding by manual pressure while in patients centrally cannulated contact the cardiac surgeon for urgent thoracotomy

-Replace the blood loss as fast as possible

-Re-establish the vascular access

-Obtain another circuit

-Clamp the circuit

-Start CPR

-Carefully evaluate the patient for systemic embolism and consider neuroprotective measures as hypothermia

$A C T$ activated clotting time, $U S$ ultrasound, $R P M$ revolutions per minutes, $R A$ right atrium, $C P R$ cardio-pulmonary resuscitation 
concomitant respiratory comorbidities, often need mechanical respiratory support in both peri- and post-operative period. Obviously, the duration and type of mechanical ventilation depend on the basal lung functions and underlying disease. The lung protective principles are useful among the different ventilation strategies adopted during VA-ECMO support. The addition of positive-end-expiratory pressure (PEEP) could be helpful in treating those patients with an afterload-dependent LV failure since its application decreases peripheral vascular residences (PVR). On the contrary, lower levels of PEEP are suggested for those subjects experiencing a preload-dependent myocardial failure [51-54]. As well known, weaning from MV and subsequent shift to spontaneous breathing can be difficult and associated with adverse hemodynamic events. Indeed, spontaneous breathing causes both hemodynamic and neuroendocrine effects: the former encompasses a decrease in intrathoracic pressure which leads to an increase in LV afterload and venous return, resulting in increased RVEDV and LVEDV, while the latter leads to an increase in the myocardial oxygen consumption due to a significant sympathetic activation. This last neuroendocrine activation is generally more pronounced in COPD patients which had a significant higher risk of complications during the weaning process $[55,56]$. Importantly, HR-PCI patients supported with ECMO represent a subgroup of patients at higher risk of ventilatory-induced lung injury (VILI). Several mechanisms have been recognized such as the alveolar strain, the occurrence of an atelectrauma, or reabsorption atelectasis. However, as generally recommended, an appropriate ventilator setting can limit the risk of alveolar overdistension or strain.

\section{General considerations for high-risk PCI patients in CCU}

\section{Analgesia and sedation}

Analgesic protocols and data for acute pain management and sedation of HR-PCI patients are scant. HR-PCI patients generally experience somatic pain at the drain and vascular cannulation sites. However, also endotracheal suctioning and mobilization are non-neglectable causes of pain during ICU stay. The administration of both analgesics and sedatives is necessary in MCS patients to provide optimal and safe care. A deeper sedation with muscle relaxants has different beneficial effects as the optimization of blood flows and gas exchange [39]. Furthermore, it is fundamental to avoid any cannula movement which may lead to dislodgements and complications as hemolysis. Pain management and sedation must begin before PCI and then must be continued after the procedure, tailored based on validated scales. Both overdoses and underdoses of medications for pain controls should be avoided. However, choosing the most adequate analgesic agent in these patients is often a challenge among the available drug armamentarium. The selection must take into account the pharmacodynamic and pharmacokinetic properties as well as the ongoing organ dysfunctions. Among the opioids, fentanyl and morphine have been reported as the most frequently administered while among sedatives, midazolam and propofol have been suggested [57]. For ECMO patients requiring high dose of opioids and sedatives during ECMO support, ketamine has been suggested as a viable alternative [58].

\section{Electrolytes and metabolic alterations}

Maintenance of electrolyte concentrations as well as fluid balance and acid-base homeostasis is vital to ensure body function. The prompt recognition of these disorders, which are frequently observed in HR-PCI patients hospitalized in ICU, is crucial to prevent further cardiac impairment or neurological complications. At the same manner, both hypovolemia and fluid overload must be avoided. In this regard, diuretic agents are doubtless helpful to assist fluid balance; however, it is important to underline that adverse events related to diuretic administration are dose dependent. Since no specific studies have yet been performed on these issues in HR-PCI patients' traditional therapeutic strategies, based on fluid administration, electrolyte substitution and in some cases to extracorporeal filtration must be adopted also in this subgroup of patients. Reviewing the available literature, Schmidt et al. have demonstrated that a positive fluid balance over the first 3 days in HR-PCI patients supported with VA-ECMO was an independent predictor of 90-day mortality [59].

\section{Drug administration}

In VA-ECMO patients, the drug dose should be optimized. Indeed, the interaction between the ECMO circuit and the physicochemical properties of several drugs may lead to significant changes in the pharmacokinetic and pharmacodynamic properties modifying the dosing requirements. Moreover, the ECMO alters the apparent volume distribution $(\mathrm{Vd})$ through the hemodilution from priming solution, drug sequestration, and ECMO-related physiological changes. These aspects must be carefully considered when antibiotics, vasoactive drugs, and diuretic agents are administered [60, 61].

\section{Acute kidney injury}

Acute kidney injury (AKI) represents a frequent and serious complication in patients admitted to CCU with severe HF. Different degrees of renal impairment can be observed in patients requiring HR-PCI. In the same manner, different underlying processes, such as nephrotoxicity, hemodynamic, neurohormonal, or inflammatory abnormalities, can be encountered in daily clinical practice [62]. Considering that traditionally monitoring of renal function, based on serum creatinine or urea, has a limited use in critically ill patients, a daily 
evaluation based on the urinary creatinine clearance or on the estimated glomerular filtration rate (eGFR) must be performed. In elective procedure, baseline renal function, evaluation of comorbidities influencing the renal filtration, as arterial hypertension, diabetes, anemia, or high dose of diuretics must be carefully evaluated to minimize AKI. A fundamental aspect is represented by a careful pre-operative assessment of renal function in those patients with pre-existing renal impairment that may be aggravated by intravenous contrast media. Contrast-induced acute kidney injury (CI-AKI) represents another critical aspect in HR-PCI patients. Ideally, adequate preoperative IV volume expansion is recommended $24 \mathrm{~h}$ before the procedure, as well as the discontinuation of drugs with some potential nephrotoxic or metabolic effects as metformin [63]. Low-contrast protocols and prophylaxis for contrast allergy must be encouraged and adopted whenever possible. Renal replacement therapy (RRT) must be adopted in lifethreatening modifications. Ostermann et al. have reviewed the most common indications for RRT during and continuous renal replacement therapy (CRRT) during ECMO. Their results suggest that the optimal timing for initiation of CRRT must be individualized, considering the degree of fluid overload and severity of AKI-related metabolic derangements. CRRT can be provided using two different approaches: using an in-line hemofilter or a fully integrated CRRT device or through a parallel system with separate ECMO and RRT circuits [64].

\section{Limitations}

The term HR-PCI as well as the therapeutic strategies adopted to treat these patients represent a relatively new field of cardiovascular medicine. A larger part of data that support the use of VA-ECMO in combination with other MCS have been obtained from studies performed on patients with CS with or without AMI. Conversely, few studies have been performed specifically in HR-PCI patients. As a result, areas of uncertainty associated with the MCS in HR-PCI still exist also in the treatment of ECMO patients after HR-PCI. Further studies performed on these patients will help to further improve the MCS during PCI. Moreover, the aim of the review was not to perform a systematic analysis on the use of VA-ECMO in HRPCI patients or a device-based review but to help physicians in the management of these complex patients which will become more frequent in the future.

\section{Conclusions}

As emerged by our review, the management of HR-PCI patients requires a holistic approach. Interventional cardiologists, clinical cardiologists, and cardiac surgeons must share their knowledge and expertise to treat these patients. Some important aspects, which are faced every day in CCU, have been poorly investigated in HR-PCI patients. In the field of HR-PCI, the role of interventional cardiologists ranges from the defining goals of care and preprocedural patient's optimization, managing concomitant severe comorbidities and provides an adequate postprocedural care. In this clinical setting, a synergic cooperation between intensivists and cardiologists may be useful to deliver a patient-individual tailored therapy to impact patients' outcomes. Hub and spoke models for HRPCI must be clearly delineated such that community cardiology centers should be able to establish efficient transfer mechanisms for those patients requiring VA-ECMO support.

\section{Compliance with ethical standards}

Conflict of interest The authors declare that they have no conflicts of interest

Ethical approval This article does not contain any studies with human participants or animals performed by any of the authors.

\section{References}

1. Atkinson TM, Ohman EM, O'Neill WW, Rab T, Cigarroa JE, Interventional Scientific Council of the American College of Cardiology (2016) A practical approach to mechanical circulatory support in patients undergoing percutaneous coronary intervention: An interventional perspective. JACC Cardiovasc Interv 9:871-883

2. Agrawal H, Aggarwal K (2016) Mechanical circulatory support in percutaneous coronary interventions: expanding the possibilities. J Invasive Cardiol 28:243-245

3. Go AS, Mozaffarian D, Roger VL, Benjamin EJ, Berry JD, Blaha MJ, Dai S, Ford ES, Fox CS, Franco S, Fullerton HJ, Gillespie C, Hailpern SM, Heit JA, Howard VJ, Huffman MD, Judd SE, Kissela BM, Kittner SJ, Lackland DT, Lichtman JH, Lisabeth LD, Mackey RH, Magid DJ, Marcus GM, Marelli A, Matchar DB, McGuire DK, Mohler ER 3rd, Moy CS, Mussolino ME, Neumar RW, Nichol G, Pandey DK, Paynter NP, Reeves MJ, Sorlie PD, Stein J, Towfighi A, Turan TN, Virani SS, Wong ND, Woo D, Turner MB, American Heart Association Statistics Committee and Stroke Statistics Subcommittee (2014) Heart disease and stroke statistics-2014 update: a report from the American Heart Association. Circulation 129:e28-e292

4. Rihal CS, Naidu SS, Givertz MM, Szeto WY, Burke JA, Kapur NK, Kern M, Garratt KN, Goldstein JA, Dimas V, Tu T, Society for Cardiovascular Angiography and Interventions (SCAI); Heart Failure Society of America (HFSA); Society for Thoracic Surgeons (STS); American Heart Association (AHA); American College of Cardiology (ACC) (2015) 2015 SCAI/ACC/HFSA/ STS Clinical expert consensus statement on the use of percutaneous mechanical circulatory support devices in cardiovascular care (Endorsed by the American Heart Association, the Cardiological Society of India, and Sociedad Latino Americana de Cardiologia Intervencion; Affirmation of value by the Canadian Association of Interventional Cardiology-Association Canadienne de Cardiologie d'intervention). J Card Fail 21:499-518

5. Craner RC, Carvajal T, Villablanca PA, Jahanyar J, Yang EH, Ramakrishna H (2018) The increasing importance of percutaneous 
mechanical circulatory support in high-risk transcatheter coronary interventions: an evidence-based analysis. J Cardiothorac Vasc Anesth 32:1507-1524

6. Guerrero-Miranda CY, Hall SA (2019) Cardiac catheterization and percutaneous intervention procedures on extracorporeal membrane oxygenation support. Ann Cardiothorac Surg 8:123-128

7. Parhar K, Millar V, Zochios V, Bruton E, Jaworksi C, West N, Vuylsteke A (2018) Clinical outcomes of patients undergoing primary percutaneous coronary intervention for acute myocardial infarction requiring the intensive care unit. J Intensive Care 6:5

8. Sianos G, Morel MA, Kappetein AP, Morice MC, Colombo A, Dawkins K, van den Brand M, Van Dyck N, Russell ME, Mohr FW, Serruys PW (2005) The SYNTAX Score: an angiographic tool grading the complexity of coronary artery disease. EuroIntervention 1:219-227

9. Serruys PW, Morice MC, Kappetein AP, Colombo A, Holmes DR, Mack MJ, Ståhle E, Feldman TE, van den Brand M, Bass EJ, Van Dyck N, Leadley K, Dawkins KD, Mohr FW, SYNTAX Investigators (2009) Percutaneous coronary intervention versus coronary-artery bypass grafting for severe coronary artery disease. N Engl J Med 360:961-972

10. Burzotta F, Trani C, Doshi SN, Townend J, van Geuns RJ, Hunziker P, Schieffer B, Karatolios K, Møller JE, Ribichini FL, Schäfer A, Henriques JP (2015) Impella ventricular support in clinical practice: collaborative viewpoint from a European expert user group. Int $\mathrm{J}$ Cardiol 201:684-691

11. Briceno N, De Silva K, Ryan M, Patterson T, O'Gallagher K, Ellis H, Rivolo S, Lee J, Redwood S, Shah AM, Marber M, Perera D (2019) Intra-aortic balloon counterpulsation for high-risk percutaneous coronary intervention: Defining coronary responders. J Cardiovasc Transl Res 12:299-309. https://doi.org/10.1007/ s12265-019-09871-8

12. Vetrovec GW (2017) Hemodynamic support devices for shock and high-risk PCI: When and which one. Curr Cardiol Rep 19:100

13. Tomasello SD, Boukhris M, Ganyukov V, Galassi AR, Shukevich D, Haes B, Kochergin N, Tarasov R, Popov V, Barbarash L (2015) Outcome of extracorporeal membrane oxygenation support for complex high-risk elective percutaneous coronary interventions: A single-center experience. Heart Lung 44:309-313

14. Hryniewicz K, Sandoval Y, Samara M, Bennett M, Cabuay B, Chavez IJ, Seatter S, Eckman P, Zimbwa P, Dunn A, Sun B (2016) Percutaneous venoarterial extracorporeal membrane oxygenation for refractory cardiogenic shock is associated with improved short- and long-term survival. ASAIO J 62:397-402

15. Keebler ME, Haddad EV, Choi CW, McGrane S, Zalawadiya S, Schlendorf KH, Brinkley DM, Danter MR, Wigger M, Menachem JN, Shah A, Lindenfeld 2 (2018) Venoarterial extracorporeal membrane oxygenation in cardiogenic shock. JACC Heart Fail 6:503-516

16. Shaukat A, Hryniewicz-Czeneszew K, Sun B, Mudy K, Wilson K, Tajti P, Stanberry L, Garberich R, Sandoval Y, Burke MN, Chavez I, Gössl M, Henry T, Lips D, Mooney M, Poulose A, Sorajja P, Traverse J, Wang Y, Bradley S, Brilakis ES (2018) Outcomes of extracorporeal membrane oxygenation support for complex highrisk elective percutaneous coronary interventions: A single-center experience and review of the literature. J Invasive Cardiol 30:456460

17. Huang CC, Hsu JC, Wu YW, Ke SR, Huang JH, Chiu KM, Liao PC (2018) Implementation of extracorporeal membrane oxygenation before primary percutaneous coronary intervention may improve the survival of patients with ST-segment elevation myocardial infarction and refractory cardiogenic shock. Int J Cardiol 269:45-50

18. Patel SM, Lipinski J, Al-Kindi SG, Patel T, Saric P, Li J, Nadeem F, Ladas T, Alaiti A, Phillips A, Medalion B, Deo S, Elgudin Y, Costa MA, Osman MN, Attizzani GF, Oliveira GH, Sareyyupoglu B, Bezerra HG (2019) Simultaneous venoarterial extracorporeal membrane oxygenation and percutaneous left ventricular decompression therapy with impella is associated with improved outcomes in refractory cardiogenic shock. ASAIO J 65:21-28

19. Pappalardo F, Schulte C, Pieri M, Schrage B, Contri R, Soeffker G, Greco T, Lembo R, Müllerleile K, Colombo A, Sydow K, De Bonis M, Wagner F, Reichenspurner H, Blankenberg S, Zangrillo A, Westermann D (2017) Concomitant implantation of Impella ${ }^{\circledR}$ on top of veno-arterial extracorporeal membrane oxygenation may improve survival of patients with cardiogenic shock. Eur J Heart Fail 19:404-412

20. Akanni OJ, Takeda K, Truby LK, Kurlansky PA, Chiuzan C, Han J, Topkara VK, Yuzefpolskaya M, Colombo PC, Karmpaliotis D, Moses JW, Naka Y, Garan AR, Kirtane AJ, Takayama H (2019) EC-VAD: Combined use of extracorporeal membrane oxygenation and percutaneous microaxial pump left ventricular assist device. ASAIO J 65:219-226

21. Chen K, Hou J, Tang H, Hu S (2019) Concurrent implantation of intra-aortic balloon pump and extracorporeal membrane oxygenation improved survival of patients with postcardiotomy cardiogenic shock. Artif Organs 43:142-149

22. Li Y, Yan S, Gao S, Liu M, Lou S, Liu G, Ji B, Gao B (2018) Effect of an intra-aortic balloon pump with venoarterial extracorporeal membrane oxygenation on mortality of patients with cardiogenic shock: a systematic review and meta-analysis. Eur J Cardiothorac Surg. https://doi.org/10.1093/ejcts/ezy304

23. Chakaramakkil MJ, Sivathasan C (2018) ECMO and short-term support for cardiogenic shock in heart failure. Curr Cardiol Rep 20:87

24. Aragon J, Lee MS, Kar S, Makkar RR (2005) Percutaneous left ventricular assist device: "TandemHeart" for high-risk coronary intervention. Catheter Cardiovasc Interv 65:346-352

25. Negi SI, Malahfji M, Sokolovic M, Torguson R, Didier R, Gregoric I, Loyalka P, Pichard A, Satler LF, Kar BO, Waksman R (2015) TCT-199 A comparative analysis of use of extracorporeal membrane oxygenation and peripheral ventricular assist device tandemheart in acute myocardial infarction. J Am Coll Cardiol 66(Supplement 1). https://doi.org/10.1016/j.jacc.2015.08.213]

26. Chamogeorgakis T, Rafael A, Shafii AE, Nagpal D, Pokersnik JA, Gonzalez-Stawinski GV (2013) Which is better: a miniaturized percutaneous ventricular assist device or extracorporeal membrane oxygenation for patients with cardiogenic shock? ASAIO J 59:607611

27. Bernhardt AM, Hillebrand M, Yildirim Y, Hakmi S, Wagner FM, Blankenberg S, Reichenspurner H, Lubos E (2018) Percutaneous left atrial unloading to prevent pulmonary oedema and to facilitate ventricular recovery under extracorporeal membrane oxygenation therapy. Interact Cardiovasc Thorac Surg 26:4-7

28. Merchán S, Martín-Moreiras J, Uribarri A, López J, Reta L, Sánchez PL (2015) Ventricular support with extracorporeal membrane oxygenation: beyond cardiogenic shock treatment. Rev Esp Cardiol (Engl Ed) 68:897-879

29. Staudacher DL, Langner O, Biever P, Benk C, Zehender M, Bode C, Wengenmayer T (2015) Unprotected left main percutaneous coronary intervention in acute coronary syndromes with extracorporeal life support backup. Scientifica (Cairo) 2015:435878. https:// doi.org/10.1155/2015/435878

30. Antoku D, Ghasem W, Mehra A, Matthews RV, Clavijo LC, Hindoyan A, Shavelle DM, Dhillon AS (2018) CRT-600.09 CardioHELP support for high-risk percutaneous coronary intervention: A single center case series. JACC Cardiovasc Interv 11(Supplement 4). https://doi.org/10.1016/j.jcin.2018.01.149

31. Chung M, Shiloh AL, Carlese A (2014) Monitoring of the adult patient on venoarterial extracorporeal membrane oxygenation. Sci World J 393258. https://doi.org/10.1155/2014/393258

32. Platts DG, Sedgwick JF, Burstow DJ, Mullany DV, Fraser JF (2012) The role of echocardiography in the management of patients 
supported by extracorporeal membrane oxygenation. J Am Soc Echocardiogr 25:131-141

33. Aissaoui N, Luyt CE, Leprince P, Trouillet JL, Léger P, Pavie A, Diebold B, Chastre J, Combes A (2011) Predictors of successful extracorporeal membrane oxygenation (ECMO) weaning after assistance for refractory cardiogenic shock. Intensive Care Med 37: 1738-1745

34. Pappalardo F, Pieri M, Arnaez Corada B, Ajello S, Melisurgo G, De Bonis M, Zangrillo A (2015) Timing and strategy for weaning from venoarterial ECMO are complex issues. J Cardiothorac Vasc Anesth 29:906-911

35. Cavarocchi NC, Pitcher HT, Yang Q, Karbowski P, Miessau J, Hastings HM, Hirose H (2013) Weaning of extracorporeal membrane oxygenation using continuous hemodynamic transesophageal echocardiography. J Thorac Cardiovasc Surg 146:1474-1479

36. Aso S, Matsui H, Fushimi K, Yasunaga H (2016) In-hospital mortality and successful weaning from venoarterial extracorporeal membrane oxygenation: analysis of 5,263 patients using a national inpatient database in Japan. Crit Care 20:80

37. Ortuno S, Delmas C, Diehl JL, Bailleul C, Lancelot A, Naili M, Cholley B, Pirracchio R, Aissaoui N (2019) Weaning from venoarterial extra-corporeal membrane oxygenation: which strategy to use? Ann Cardiothorac Surg 8:E1-E8

38. Aziz TA, Singh G, Popjes E, Stephenson E, Mulvey S, Pae W, ElBanayosy A (2010) Initial experience with CentriMag extracorporal membrane oxygenation for support of critically ill patients with refractory cardiogenic shock. J Heart Lung Transplant 29:66-71

39. ELSO Guidelines for Cardiopulmonary Extracorporeal Life Support Extracorporeal Life Support Organization, Version 1.3 November 2013 Ann Arbor, MI, USA. Available online: http:// www.elsonet.org

40. Aissaoui N, El-Banayosy A, Combes A (2015) How to wean a patient from veno-arterial extracorporeal membrane oxygenation. Intensive Care Med 41:902-905

41. Huang KC, Lin LY, Chen YS, Lai CH, Hwang JJ, Lin LC (2018) Three-dimensional echocardiography-derived right ventricular ejection fraction correlates with success of decannulation and prognosis in patients stabilized by venoarterial extracorporeal life support. J Am Soc Echocardiogr 31:169-179

42. Belohlavek J, Rohn V, Jansa P, Tosovsky J, Kunstyr J, Semrad M, Horak J, Lips M, Mlejnsky F, Balik M, Klein A, Linhart A, Lindner $\mathrm{J}$ (2010) Veno-arterial ECMO in severe acute right ventricular failure with pulmonary obstructive hemodynamic pattern. J Invasive Cardiol 22:365-369

43. Rao P, Khalpey Z, Smith R, Burkhoff D, Kociol RD (2018) Venoarterial extracorporeal membrane oxygenation for cardiogenic shock and cardiac arrest. Circ Heart Fail 11:e004905

44. Kajimoto M, Ledee DR, Isern NG, Portman MA (2017) Right ventricular metabolism during venoarterial extracorporeal membrane oxygenation in immature swine heart in vivo. Am J Physiol Heart Circ Physiol 312:H721-H727

45. Cheng R, Hachamovitch R, Kittleson M, Patel J, Arabia F, Moriguchi J, Esmailian F, Azarbal B (2014) Complications of extracorporeal membrane oxygenation for treatment of cardiogenic shock and cardiac arrest: a meta-analysis of 1,866 adult patients. Ann Thorac Surg 97:610-616

46. Yen CC, Kao CH, Tsai CS, Tsai SH (2018) Identifying the risk factor and prevention of limb ischemia in extracorporeal membrane oxygenation with femoral artery cannulation. Heart Surg Forum 21: E018-E022

47. Makdisi G, Makdisi T, Wang IW (2017) Use of distal perfusion in peripheral extracorporeal membrane oxygenation. Ann Transl Med 5:103. https://doi.org/10.21037/atm.2017.03.01
48. Mulder MMG, Hassan I, Lancé M (2018) ECMO and anticoagulation: A comprehensive review. Netherlands. J Crit Care 26:6-13

49. Wong J, Lam J, Mok Y, Lee J (2018) Anticoagulation in extracorporeal membrane oxygenation. JECCM 2:12. https://doi.org/10. 21037/jeccm.2018.01.12

50. Hamed A, Alinier G, Hassan IF (2018) The ECMO specialist's role in troubleshooting ECMO emergencies. Egypt J Crit Care Med 6: 91-93. https://doi.org/10.1016/j.ejccm.2018.12.011

51. Schmidt M, Pellegrino V, Combes A, Scheinkestel C, Cooper DJ, Hodgson C (2014) Mechanical ventilation during extracorporeal membrane oxygenation. Crit Care 18:203

52. Duke GJ (1999) Cardiovascular effects of mechanical ventilation Crit Care Resusc 1:388-399

53. Jardin F, Vieillard-Baron A (2007) Is there a safe plateau pressure in ARDS? The right heart only knows. Intensive Care Med 33:444 447

54. Chen WL, Chen GY, Kuo CD (2006) Hypoxemia and autonomic nervous dysfunction in patients with chronic obstructive pulmonary disease. Respir Med 100:1547-1553

55. Ahmed SM, Athar M (2015) Mechanical ventilation in patients with chronic obstructive pulmonary disease and bronchial asthma. Indian J Anaesth 59:589-598

56. Hickling KG (2001) Best compliance during a decremental, but not incremental, positive end-expiratory pressure trial is related to open-lung positive end-expiratory pressure: a mathematical model of acute respiratory distress syndrome lungs. Am J Respir Crit Care Med 163:69-78

57. Marhong JD, DeBacker J, Viau-Lapointe J, Munshi L, Del Sorbo L, Burry L, Fan E, Mehta S (2017) Sedation and Mobilization During Venovenous Extracorporeal Membrane Oxygenation for Acute Respiratory Failure: An International Survey. Crit Care Med 45: 1893-1899

58. Maybauer MO, Koerner MM, Maybauer DM (2019) Perspectives on adjunctive use of ketamine for analgosedation during extracorporeal membrane oxygenation. Expert Opin Drug Metab Toxicol $26: 1-3$

59. Schmidt M, Bailey M, Kelly J, Hodgson C, Cooper DJ, Scheinkestel C, Pellegrino V, Bellomo R, Pilcher D (2014) Impact of fluid balance on outcome of adult patients treated with extracorporeal membrane oxygenation. Intensive Care Med 40: $1256-1266$

60. Cheng V, Abdul-Aziz MH, Roberts JA, Shekar K (2018) Optimising drug dosing in patients receiving extracorporeal membrane oxygenation. J Thorac Dis 10:S629-S641

61. Dzierba AL, Abrams D, Brodie D (2017) Medicating patients during extracorporeal membrane oxygenation: the evidence is building. Crit Care 21:66

62. Tarvasmäki T, Haapio M, Mebazaa A, Sionis A, Silva-Cardoso J, Tolppanen H, Lindholm MG, Pulkki K, Parissis J, Harjola VP, Lassus J, CardShock Study Investigators (2018) Acute kidney injury in cardiogenic shock: definitions, incidence, haemodynamic alterations, and mortality. Eur J Heart Fail 20:572-581

63. Yang Y, George KC, Luo R, Cheng Y, Shang W, Ge S, Xu G (2018) Contrast-induced acute kidney injury and adverse clinical outcomes risk in acute coronary syndrome patients undergoing percutaneous coronary intervention: a meta-analysis. BMC Nephrol 19:374

64. Ostermann M, Connor M Jr, Kashani K (2018) Continuous renal replacement therapy during extracorporeal membrane oxygenation: why, when and how? Curr Opin Crit Care 24:493-503

Publisher's note Springer Nature remains neutral with regard to jurisdictional claims in published maps and institutional affiliations. 